Check for updates

Cite this: RSC Adv., 2017, 7, 56550

\title{
Fabrication of aqueous-based dual drug loaded silk fibroin electrospun nanofibers embedded with curcumin-loaded RSF nanospheres for drugs controlled release $\uparrow$
}

\author{
Huijun Li, (D) + Jingxin Zhu, (D) * Song Chen, Lan Jia and Yanlong Ma
}

This paper presents a new nanofabrication method for dual drug loaded regenerated silk fibroin (RSF) nanofibers, based on a simple, colloid-electrospinning technique. In this process, curcumin (CUR, hydrophobic drug), loaded in RSF nanospheres, and doxorubicin hydrochloride (DOX. $\mathrm{HCl}$, hydrophilic drug) were blended with aqueous-based RSF solutions to prepare the nanofibers embedded with CURloaded nanospheres in core and $\mathrm{DOX} \cdot \mathrm{HCl}$ in shell. The dual drug loaded nanofibers showed smooth surfaces and relatively uniform diameters and dispersity. The encapsulation of CUR-loaded nanospheres and $\mathrm{DOX} \cdot \mathrm{HCl}$ were confirmed by electron and fluorescence microscopy. FTIR spectra and X-ray diffraction results indicated that presence of intermolecular hydrogen bonding between drug and RSF fibers and the drugs were presented in an amorphous state in the nanofibers. In vitro dissolution tests showed that CUR-loaded nanospheres/DOX.HCl-loaded RSF core/shell nanofibers exhibited dual drug release profiles and achieved a sustained release, furthermore, the amount of drug release in core or shell phase of nanofibers was tunable by controlling the drug-loaded content and crystal content of nanofibers with water-annealing process at different temperature. The release kinetics study showed that the mechanism of drug release could be described by a Fickian model. This drug delivery system could be potentially used as local multi-drug delivery systems for treatment of several medical conditions, including breast cancer or skin cancer.

Received 13th November 2017 Accepted 8th December 2017

DOI: 10.1039/c7ra12394a

rsc.li/rsc-advances by electrospinning SF mixtures with various therapeutic agents; the treatment efficacy of therapeutic agent is often limited. An increasing number of studies have demonstrated that multidrug loading and the programmable release of each drug are important in tissue engineering and disease therapy. ${ }^{10-12}$ Liu et al. incorporated dual growth factors into regenerated silk fibroin (RSF) electrospun nanofiber scaffolds and found that the scaffolds significantly promoted nerve regeneration when it was implanted into mice. ${ }^{13}$ However, the fabrication of the multidrug loading nanofibers is quite challenging task. It needs to consider the influences of various factors, such as the solubility of drug, the volatility of solvent, the interactions between drug and polymer and suitable the electrospinning process. Recently, the nanofibers with core-shell structure formed by coaxial electrospinning process or colloid-electrospinning technique can result in successful incorporation of several active substances into nanofibers. ${ }^{14-16}$ Nevertheless, coaxial electrospinning is affected by several parameters, such as the solubility of drugs in polymer solutions, the interactions between the core and shell polymer solutions and their flow rates, and it do not necessarily result in core-shell nanofibers. ${ }^{17-19}$ The colloidelectrospinning technique is more favorable than coaxial spinning, because a simple single-nozzle electrospinning process
College of Materials Science and Engineering, Taiyuan University of Technology, Taiyuan, 030024, P. R. China.E-mail: zhujingxin@tyut.edu.cn

$\dagger$ Electronic supplementary information (ESI) available. See DOI: 10.1039/c7ra12394a

\$ H. Li and J. Zhu contributed equally to this work. 
and easy blend nano- or microcapsules or small emulsion droplets in colloidal dispersion solution. This method can produce nanofibers embedded with nanocontainers which can be loaded catalysts, dyes or drugs. ${ }^{20-22}$

Curcumin (CUR), a potent bioactive agent, was found in turmeric exhibiting a variety of good properties like antioxidant, anti-inflammatory, anticancer. ${ }^{\mathbf{2 3 2 4}}$ But its clinical application is currently limited, due to its low bioavailability and poor solubility in aqueous media. Doxorubicin hydrochloride $(\mathrm{DOX} \cdot \mathrm{HCl})$ is hydrophilic antitumor and anticancer drugs. It was found that the two drugs could achieve synergistic therapeutic effect in cancer therapy. ${ }^{25,26}$

In this paper, we developed a new and convenient method to prepare dual drug loaded silk fibroin electrospun nanofibers embedded with CUR-loaded RSF nanospheres in all aqueous system. In brief, firstly, CUR, hydrophobic drug, was loaded in SF nanospheres by ethanol precipitation the self-assembly of silk protein, then the CUR-loaded SF nanospheres and $\mathrm{DOX} \cdot \mathrm{HCl}$, hydrophilic drug, were blended with RSF aqueous solutions to produce nanofibers through a colloidelectrospinning technique. The encapsulation of CUR-loaded nanospheres in core phase and $\mathrm{DOX} \cdot \mathrm{HCl}$ in shell phase of the nanofibers were examined by electron and fluorescence microscopy. By controlling the amount of drug-loaded and crystal content of RSF matrix, we achieved the purpose of control the dual drug release rate. And the drug release kinetics was further studied.

\section{Materials and methods}

\section{Chemicals and regents}

Cocoons of Bombyx mori were purchased from Zhejiang, China. Tris(hydroxymethyl)aminomethane (tris), 2-( $N$-morpholine) ethyl sulfonic acid (MES), curcumin (CUR, >99\%) and doxorubicin hydrochloride (DOX $\cdot \mathrm{HCl},>98 \%$ ) were purchased from Sangon Biotech Co., Ltd (Shanghai, China). All other chemicals, including $\mathrm{LiBr}, \mathrm{Na}_{2} \mathrm{CO}_{3}$ and ethanol were analytical grade and used without further purification.

\section{Preparation of pure regenerated silk fibroin (RSF) aqueous solution}

RSF aqueous solution was prepared using previously described procedure. ${ }^{27}$ Briefly, cocoons of Bombyx mori were degummed twice with $0.5 \mathrm{wt} \% \mathrm{Na}_{2} \mathrm{CO}_{3}$ solution at $100{ }^{\circ} \mathrm{C}$ for $30 \mathrm{~min}$ and washed with deionized water to remove the sericin. After drying at room temperature, the degummed fibers were dissolved in a $9.3 \mathrm{~mol} \mathrm{~L}{ }^{-1} \mathrm{LiBr}$ solution at $40{ }^{\circ} \mathrm{C}$ for $2 \mathrm{~h}$. This solution was dialyzed in deionized water for 3 days with a cellulose semipermeable membrane (molecular weight cut off $14000 \pm$ $2000 \mathrm{Da}$ ), yield a solution about $2 \mathrm{wt} \%$. Then, the solution was condensed to $20 \mathrm{wt} \%$ by forced airflow at $15{ }^{\circ} \mathrm{C}$.

\section{Preparation of single and dual drug loaded RSF electrospun solutions}

CUR-loaded silk fibroin nanospheres were prepared by ethanol precipitation the self-assembly of silk protein method according to the literatures reported previously. ${ }^{28}$ The methods for detailed preparation are referred to Appendix ESI S1. $\dagger$ The preparation procedure of dual drug loaded RSF electrospun solution as follows: first, the lyophilized CUR-loaded nanospheres were dispersed in a 0.1 M MES-Tris buffer solution at pH 6.0; doxorubicin hydrochloride (DOX $\cdot \mathrm{HCl}$ ) was fully dissolved in DI water; second, the suspension of CUR-loaded nanospheres and $\mathrm{DOX} \cdot \mathrm{HCl}$ solution were added simultaneously to a $20 \mathrm{wt} \% \mathrm{RSF}$ aqueous solution at the weight ratio of CUR-loaded nanospheres to $\mathrm{RSF} 1 \mathrm{wt} \%, 3 \mathrm{wt} \%$ and $5 \mathrm{wt} \%$ and $\mathrm{DOX} \cdot \mathrm{HCl}$ to $\mathrm{RSF} 0.1 \mathrm{wt} \%$, respectively; final, the mixed solution was placed on a shaker for intensive mixing and sequentially concentrated to $33 \mathrm{wt} \%$ by airflow as a dual drug loaded electrospun solution. A single drug loaded RSF electrospun solution was prepared using the same protocol, without adding $\mathrm{DOX} \cdot \mathrm{HCl}$.

\section{Electrospinning for single and dual drug loaded RSF nanofibers}

The electrospinning process is illustrated in Fig. 1. The dual drug loaded electrospun solution was transformed into a $10 \mathrm{~mL}$ syringe capped with a steel needle (ID $=0.51 \mathrm{~mm}$ ) as a spinneret. The electrospinning was performed by applying a voltage of $30 \mathrm{kV}$, a flow rate of $0.9 \mathrm{~mL} \mathrm{~h}^{-1}$, and the distance between the tip of needle and the collector is $15 \mathrm{~cm}$. The optimization of electrospinning parameters is referred to Appendix ESI S3. $\dagger$ All the electrospinning processes were carried out at around $23 \pm$ $3{ }^{\circ} \mathrm{C}$ and $45 \pm 5 \%$ humidity.

\section{Water-annealing of electrospun RSF nanofibrous mats}

Water-annealing process was applied to increase water-insoluble of electrospun nanofibrous mats. The drug-loaded RSF nanofibrous mats were peeled off from the aluminum foil and were cut into $2 \mathrm{~cm} \times 2 \mathrm{~cm}$ small pieces. Then the samples were placed in a vacuum oven, which a water-filled plate in the bottom chamber, at 45 and $60{ }^{\circ} \mathrm{C}$ for $24 \mathrm{~h}$ at $90 \% \mathrm{RH}$. Finally, the samples were stored in a desiccator for characterization and drug release.

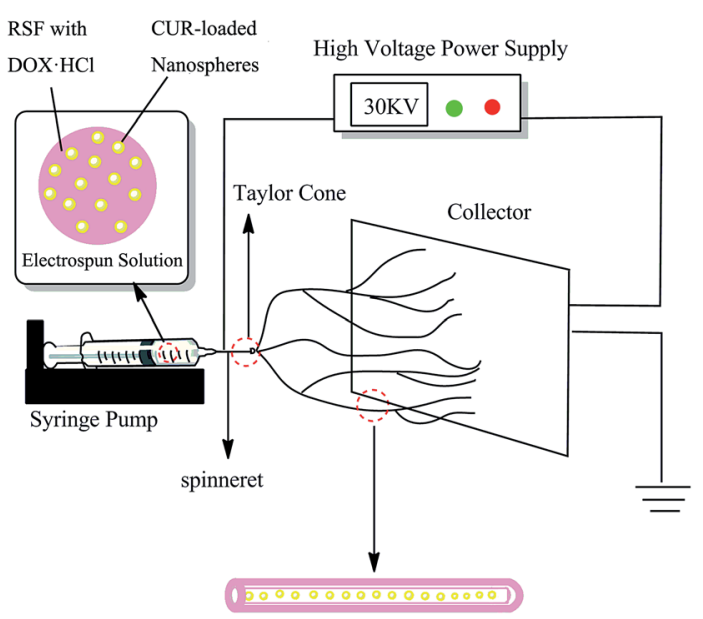

Fig. 1 Schematic drawing of electrospinning process for fabricating dual drug loaded RSF electrospun nanofibers embedded CUR-loaded nanospheres. 


\section{Characterization}

The morphology of drug loaded nanofibers was observed by using a Mira3 scanning electron microscopy (SEM) (TESCAN, Czech). Fiber diameters were measured using microstructure measurement software. The average size of CUR-loaded nanospheres were measured from the nanospheres suspension by dynamic light scattering (DLS) using a Zetasizer Nano ZS90 particle size analyzer (Malvern, England). The encapsulation and distribution of CUR-loaded nanospheres and DOX $\cdot \mathrm{HCl}$ in the nanofibers were observed by using an Axio Scope A1 fluorescence microscope (ZEISS, Germany). The embedded structure of CUR-loaded nanospheres was further characterized using a JEM-2010 high resolution transmission electron microscope (HRTEM) (JEOL, Japan). The contact angles of the electrospun nanofibrous mats treated before and after waterannealing at different temperature were measured on the JC2000D1 contact angle measuring instrument (Shanghai powereach, Chain). The average contact angles were obtained by measuring each sample three times. The interaction between drugs and RSF mats, and the structural changes of drugs-loaded electrospun nanofibrous mats before and after water-annealing, were collected on the TENSOR II Fourier-transform infrared spectrometer (Bruker, Germany) with a resolution of $4 \mathrm{~cm}^{-1}$ at the wave number range of $400-4000 \mathrm{~cm}^{-1}$. The drug distribution in the nanofibers and the structural changes of drugsloaded electrospun nanofibrous mats before and after waterannealing were obtained on a Y2000 X-ray diffraction (Dandong, China) with $\mathrm{Cu} \mathrm{K} \alpha$ radiation in the $2 \theta$ range of $5-50$ at 30 $\mathrm{kV}$ and $100 \mathrm{~mA}$, scanning speed of $3.6^{\circ} \mathrm{min}^{-1}$. Calculation of the crystallinity $(I(\%)): I(\%)=\left(A_{\mathrm{i}} / A\right) \times 100 \%$ where: $A_{\mathrm{i}}=$ area of crystalline regions, $A=$ area of all crystalline and amorphous regions. ${ }^{29}$ The data analysis was carried out by the peak separation processing software PeakFit v4.12.

\section{In vitro drug release}

The release studies for all nanofibrous mat samples with the weight of $200 \mathrm{mg}$ were immersed in the beakers containing $100 \mathrm{~mL}$ of release medium. The release medium was a mixture of phosphate buffer saline (PBS, pH 7.4) and ethanol (i.e. $5: 5 \mathrm{v}$ / $\mathrm{v}$ ratio) as a more hydrophobic component. The solutions with samples were shaken at $100 \mathrm{rpm}$ on a DKZ-2 orbital rotator (YIHENG, China) at $37{ }^{\circ} \mathrm{C}$. At predetermined time points, $4 \mathrm{~mL}$ of this solution was taken out, and $4 \mathrm{~mL}$ fresh buffer solution was added to maintain the same total solution volume. The concentration of CUR and $\mathrm{DOX} \cdot \mathrm{HCl}$ were measured by using a SQ-2800 UV-spectrophotometer (UNICO, China) at $425 \mathrm{~nm}$ and $500 \mathrm{~nm}$, respectively. The cumulative percentage of drug release was determined from a standard calibration curve. All release studies were carried out in triplicate, and results were reported as mean \pm S.D.

\section{Analysis of drug release modelling}

In order to understand drug-release mechanisms of drugloaded nanofibers, the drug release profiles were analyzed with the following Korsmeyer-Peppas eqn (1):

$$
M_{t} / M_{\infty}=k t^{n},
$$

where $M_{t}$ and $M_{\infty}$ are the cumulative amount of drug released at time $t$ and infinite time, respectively. $k$ is constant specific to the drug delivery system, $t$ is the release time, and $\mathrm{n}$ is the release exponent. ${ }^{30}$ The values of $k$ and $n$ can be obtained from a linear fit to the plot of $\log \left(M_{t} / M_{0}\right) v s . \log t$.

\section{Results and discussion}

\section{The characterization of CUR-loaded nanospheres}

The morphology and drug loading of CUR-loaded nanospheres were observed by scanning electron microscopy (SEM) and fluorescence microscope. The size of CUR-loaded nanospheres was measured by dynamic light scattering (DLS). Results are presented in Fig. 2. The CUR-loaded nanospheres were spherical granules with a relative smooth surface; the hydrophobic CUR was loaded in the core of RSF nanospheres by hydrophobic interaction with the hydrophobic segments of silk fibroin. The average diameter of the CUR-loaded nanospheres were $273.3 \pm 2 \mathrm{~nm}$. The encapsulation efficiency and drug loading of CUR in CUR-loaded nanospheres were $40.3 \%$ and $1.2 \%$ (Appendix ESI S2 $\dagger$ ).

\section{The morphology analysis of drug loaded RSF nanofibers}

In the next step, the CUR-loaded nanospheres and DOX $\cdot \mathrm{HCl}$ were mixed with RSF solution for the preparation of drugloaded nanofibers. The morphology of drug-loaded nanofibers was observed by SEM and results are presented in Fig. 3. The SEM images with the overall views of the fibers showed relatively smooth and uniform nanofibers. The average diameter of single drug loaded fibers embedded with $3 \mathrm{wt} \%$ CUR-loaded nanospheres was 1224 nm (Fig. 3A(a)). Moreover, the average diameter of dual drug loaded fibers, which embedded $1 \mathrm{wt} \%$, $3 \mathrm{wt} \%$, $5 \mathrm{wt} \%$ CUR-loaded nanospheres and added $0.1 \mathrm{wt} \% \mathrm{DOX} \cdot \mathrm{HCl}$, were 982, 987 and $1297 \mathrm{~nm}$ respectively (Fig. 3B(b), C(c) and D(d)). With increasing CURloaded nanospheres content, the fiber diameter and distribution became larger and broader. Note that in all cases the fiber diameters were at least three times larger than those of CUR-loaded nanospheres. In addition, compared Fig. 3A(a) with Fig. 3C(c), it can be found that the incorporation of $\mathrm{DOX} \cdot \mathrm{HCl}$ in the fiber makes the fiber diameters smaller. The possible reason is that adding $\mathrm{DOX} \cdot \mathrm{HCl}$ in the solution decreased the $\mathrm{pH}$ value of dual drug-loaded solution and
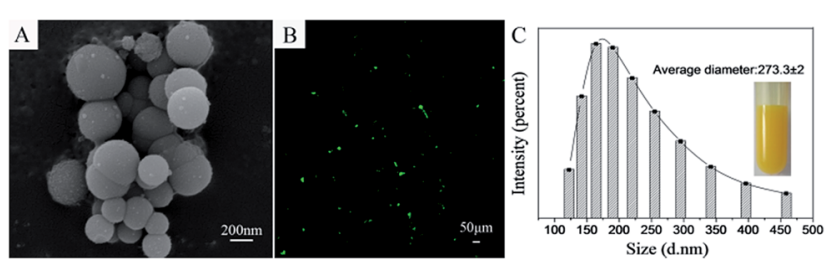

Fig. 2 SEM image, fluorescence image and DLS image of CUR-loaded nanospheres. 

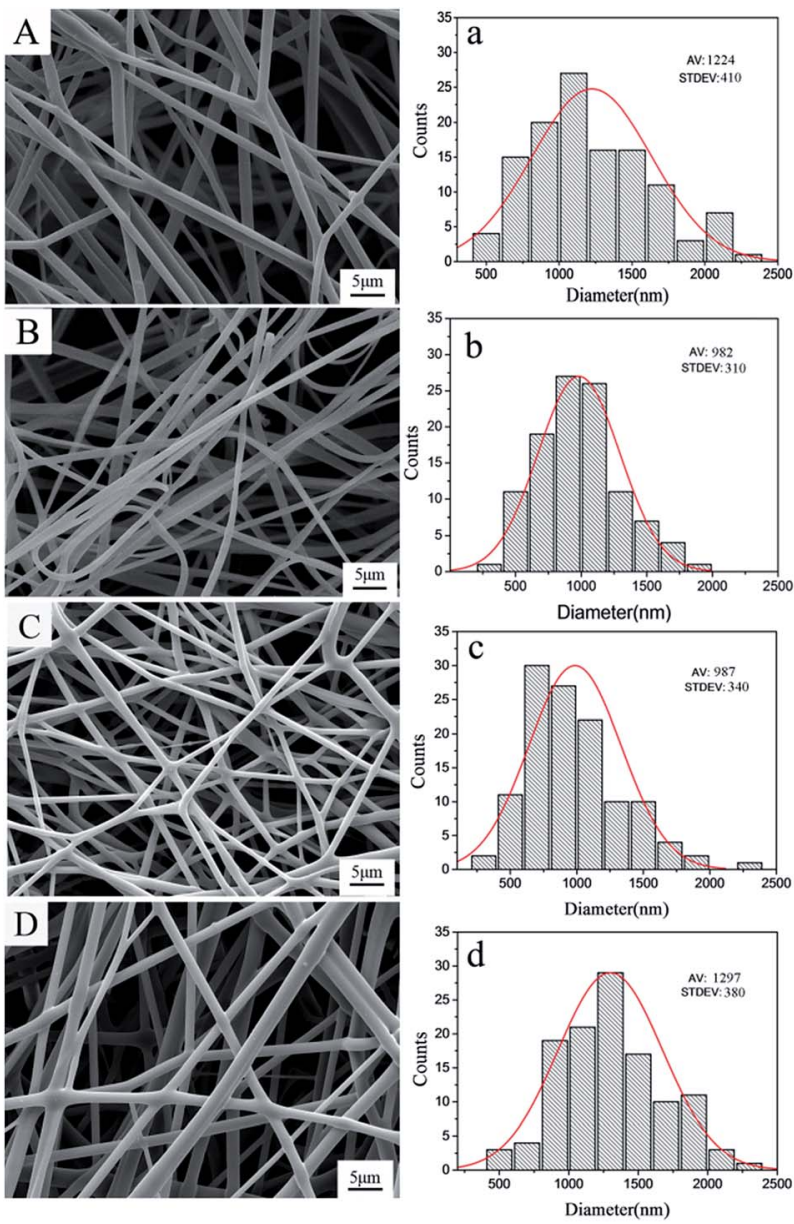

Fig. 3 The morphology and the diameter distribution of drug loaded RSF fibers: (A and a) RSF nanofibers embedded with 3 wt\% CUR-loaded nanospheres, (B and b) RSF nanofibers embedded with 1 wt\% CURloaded nanospheres in core and $0.1 \% \mathrm{DOX} \cdot \mathrm{HCl}$ in shell, (C and $\mathrm{C}$ ) RSF nanofibers embedded with 3 wt\% CUR-loaded nanospheres in core and $0.1 \% \mathrm{DOX} \cdot \mathrm{HCl}$ in shell ( $\mathrm{D}$ and d) RSF nanofibers embedded with 5 wt $\%$ CUR-loaded nanospheres in core and $0.1 \% \mathrm{DOX} \cdot \mathrm{HCl}$ in shell.

promoted the evaporation of water from the solutions, which resulted in the decrease of fiber diameter. ${ }^{27}$

\section{The structures characterization of drug loaded RSF nanofibers}

The structures of drug-loaded RSF nanofibers embedded with CUR-loaded nanospheres were observed by TEM and fluorescence microscope (Fig. 4). The CUR-loaded nanospheres were embedded in the nanofiber (Fig. 4A), the green fluoresce came from CUR-loaded nanospheres and red fluoresce came from DOX·HCl. Fig. 4B shows fluorescent image of single drug loaded nanofibers embedded with CUR-loaded nanospheres, it indicates that CUR-loaded nanospheres were encapsulated in nanofibers. Fig. 4C and D shows dual drug loaded nanofibers composited with DOX.HCl and CURloaded nanospheres at the same position of nanofiber mats. It is confirm that $\mathrm{DOX} \cdot \mathrm{HCl}$ shell phase (4C) and CUR-loaded nanospheres core phase (4D) were dispersed and encapsulated in nanofibers respectively.

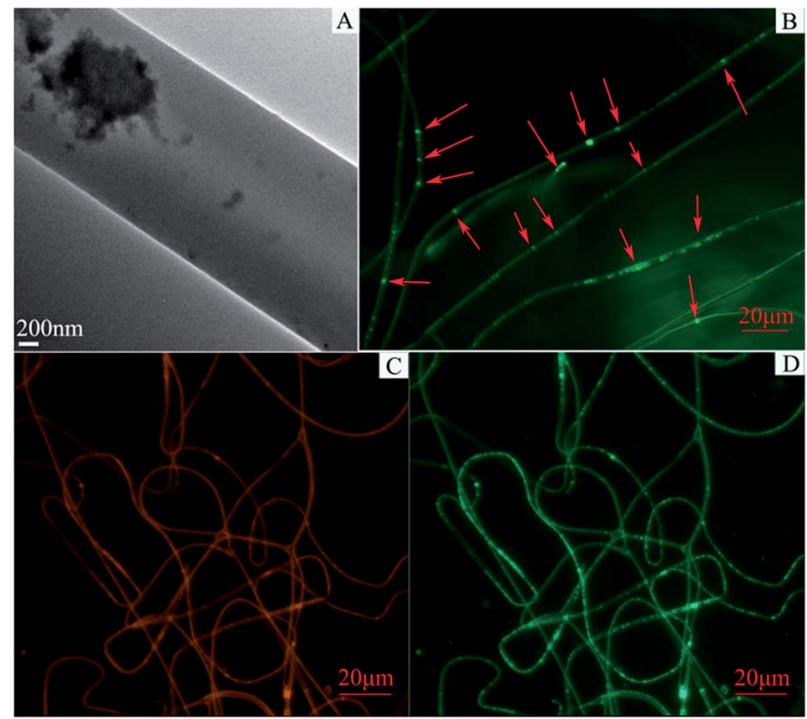

Fig. 4 TEM image and fluorescence images of drug loaded RSF nanofibers embedded with CUR-loaded nanospheres: (A) TEM image of RSF nanofibers embedded with CUR-loaded nanospheres; (B) fluorescence image of single drug loaded nanofibers embedded with CUR-loaded nanospheres; ( $C$ and D) fluorescence images of dual drug loaded nanofibers embedded with CUR-loaded nanospheres in core and $\mathrm{DOX} \cdot \mathrm{HCl}$ in shell at the same position of nanofiber mats, respectively.

The contact angle of drug loaded RSF nanofibrous mats before and after water-annealing

The size of the contact angle is an effective method for evaluation of hydrophilic and hydrophobic materials. After waterannealing, the water-insoluble and strength of RSF nanofibrous mats were enhanced. ${ }^{31}$ The hydrophobic properties changes of RSF nanofibrous mats could reflect conformation changed from the macroscopic. The results are showed in Fig. 5. Before the treatment, the contact angle was $79.0^{\circ}$, after water-annealing, the contact angle increases from $106.5^{\circ}$ to $111^{\circ}$ with the treatment temperature increasing from 45 to $60{ }^{\circ} \mathrm{C}$.

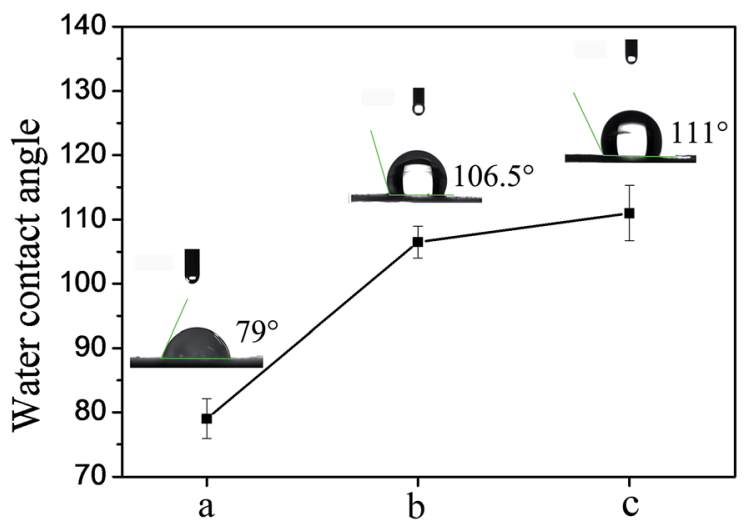

Fig. 5 The contact angle of dual drug loaded RSF nanofibrous mats before and after water-annealing at different temperature: (a) untreatment, (b) treatment at $45^{\circ} \mathrm{C}$, (c) treatment at $60{ }^{\circ} \mathrm{C}$. 

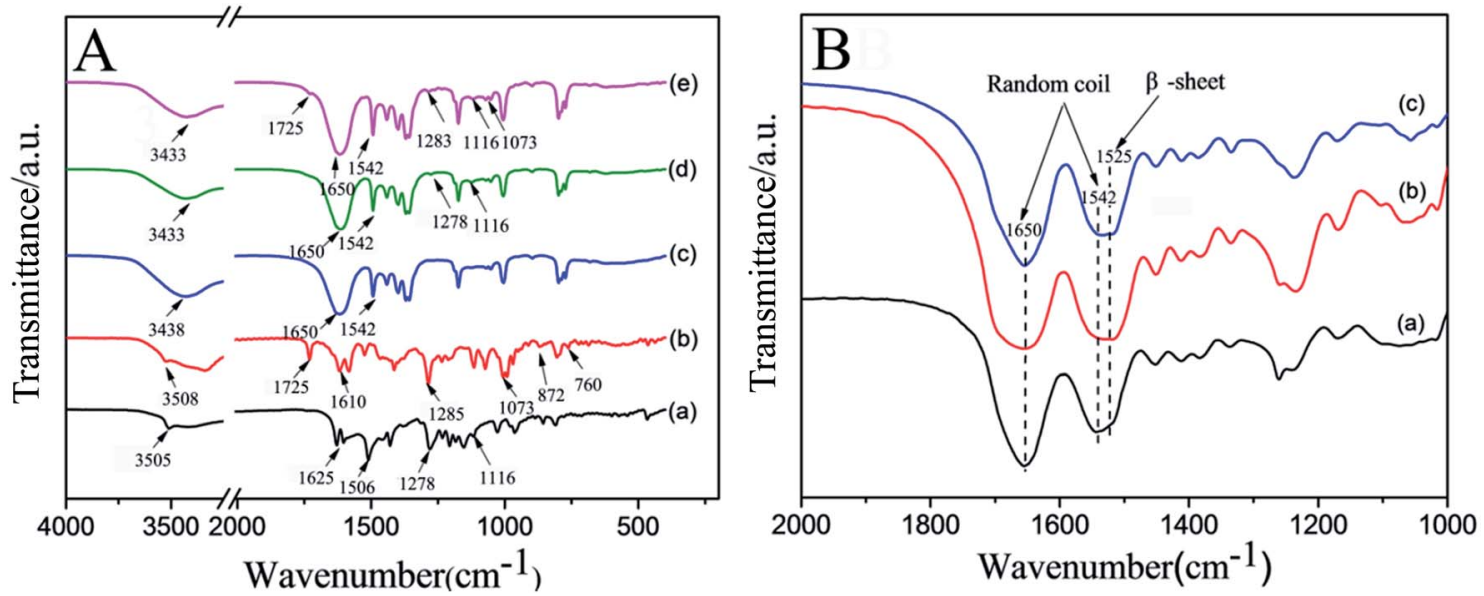

Fig. 6 (A) FTIR spectra of different materials: (A) (a) CUR, (b) DOX.HCl, (c) pure RSF nanofibers, (d) RSF nanofibers embedded with CUR-loaded nanospheres, (e) RSF nanofibers embedded with CUR-loaded nanospheres in core and DOX. $\mathrm{HCl}$ in shell; (B) FTIR spectra of dual drug loaded RSF nanofibers before and after water-annealing at different temperature: (a) untreatment, (b) treatment at $45^{\circ} \mathrm{C}$, (c) treatment at $60{ }^{\circ} \mathrm{C}$.

\section{FTIR spectral analysis}

FTIR is used to characterize the intermolecular interactions of physical mixture and the secondary structures of RSF nanofibers. The spectra of CUR, DOX $\cdot \mathrm{HCl}$, pure RSF nanofibers, RSF nanofibers embedded with CUR-loaded nanospheres, and RSF nanofibers embedded with CUR-loaded nanospheres in core and DOX $\cdot \mathrm{HCl}$ in shell are shown in Fig. 6A. The FTIR spectra of CUR is Fig. 6A(a), the characteristic peaks at 3505, 1625, 1506, 1278 and $1116 \mathrm{~cm}^{-1}$ can be ascribed to the stretching vibration of $\mathrm{O}-\mathrm{H}$ bands, $\mathrm{C}=\mathrm{O}$ bonds, $\mathrm{C}=\mathrm{C}$ bonds, $\mathrm{C}-\mathrm{O}$ bonds and $\mathrm{C}-\mathrm{O}-\mathrm{C}$ bonds, respectively. ${ }^{32,33}$ Fig. $6 \mathrm{~A}(\mathrm{~b})$ is the FTIR spectroscopy of $\mathrm{DOX} \cdot \mathrm{HCl}$, the characteristic peak at $3508,1725,1610,1285$ and $1073 \mathrm{~cm}^{-1}$ can be ascribed to the stretching vibration of $\mathrm{OH}$ bands, $\mathrm{C}=\mathrm{O}$ bonds, $\mathrm{C}=\mathrm{C}$ bonds, $\mathrm{C}-\mathrm{O}$ bonds and $\mathrm{C}-\mathrm{O}-\mathrm{C}$ bonds, the small peaks at 872 and $760 \mathrm{~cm}^{-1}$ are due to the primary amine $\mathrm{NH}_{2}$ wag and $\mathrm{N}-\mathrm{H}$ deformation bonds, respectively. ${ }^{34-36}$ Fig. $6 \mathrm{~A}(\mathrm{c})$ is the FTIR spectroscopy of pure RSF nanofibers, the characteristic peak at 1650 and $1542 \mathrm{~cm}^{-1}$ can be ascribed to the stretching vibration of amide I and amide II of RSF nanofibers. Fig. 6A(d and e) are CUR-loaded nanospheres RSF nanofibers and CUR-loaded nanospheres/DOX $\cdot \mathrm{HCl}$ core/shell RSF nanofibers, respectively.
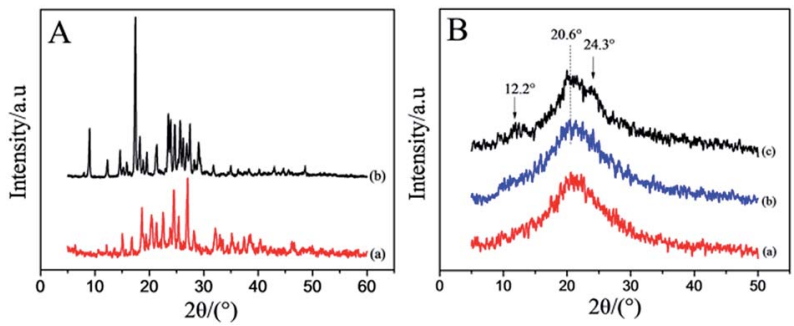

Fig. 7 The XRD of samples: (A) DOX. $\mathrm{HCl}$ (a) and CUR (b); (B) dual drug loaded RSF nanofibers before and after water-annealing at different temperature: (a) untreatment (b) treatment at $45^{\circ} \mathrm{C}$ (c) treatment at $60{ }^{\circ} \mathrm{C}$.
Compared with Fig. $6 \mathrm{~A}(\mathrm{a}-\mathrm{c})$, it was found that there were several drug related weak peaks in Fig. $6 \mathrm{~A}(\mathrm{~d}$ and e), the characteristic peaks of CUR were detected in 1278 and $1116 \mathrm{~cm}^{-1}$ (Fig. 6A(d)), suggesting that CUR-loaded nanospheres existed in RSF nanofibers; the characteristic peaks of CUR and DOX $\cdot \mathrm{HCl}$ were detected in 1725, 1283, 1116 and $1073 \mathrm{~cm}^{-1}$ (Fig. 6A(e)), indicating that $\mathrm{CUR} / \mathrm{DOX} \cdot \mathrm{HCl}$ were effective loaded in $\mathrm{RSF}$ nanofibers. In addition, there was a broad peak at $3433 \mathrm{~cm}^{-1}$ in Fig. $6 \mathrm{~A}(\mathrm{~d}$ and $\mathrm{e})$, which was a shift to the lower band of the stretching vibration of $\mathrm{OH}$ bands of CUR and $\mathrm{DOX} \cdot \mathrm{HCl}$ at 3505 and $3508 \mathrm{~cm}^{-1}$, meanwhile, in the fingerprint regions, the small peaks at 872 and $760 \mathrm{~cm}^{-1}$ of $\mathrm{DOX} \cdot \mathrm{HCl}$ almost disappeared from the spectra of drug loaded nanofibers, indicating that the presence of intermolecular hydrogen bonding between drugs and RSF matrix.

Fig. $6 \mathrm{~B}$ is the FTIR spectra of drug loaded RSF nanofibrous mats before and after water-annealing with different temperature. Before the treatment (Fig. 6B(a)), the RSF nanofibrous mats showed peaks around $1650 \mathrm{~cm}^{-1}$ (amide I) and $1542 \mathrm{~cm}^{-1}$ (amide II), indicating the RSF nanofibrous mats were dominated by random coil conformation. After water-annealing at temperature of 45 and $60{ }^{\circ} \mathrm{C}$, the peak of amide I at $1650 \mathrm{~cm}^{-1}$ basically unchanged, but the peak of amide II at $1542 \mathrm{~cm}^{-1}$ was

Table 1 The contents of the amorphous and the crystalline of RSF nanofibers before and after water-annealing at different temperature ${ }^{a}$

\begin{tabular}{lllll}
\hline & \multicolumn{4}{l}{$I(\%)=\left(A_{\mathrm{i}} / A\right) \times 100 \%$} \\
\cline { 3 - 5 } The angle of structure & Untreatment & $45{ }^{\circ} \mathrm{C}$ & $60{ }^{\circ} \mathrm{C}$ \\
\hline Silk II ( $\beta$-sheet) & $20.6^{\circ}$ & 4.93 & 25.87 & 18.17 \\
Silk II ( $\beta$-sheet) & $24.3^{\circ}$ & 0 & 0 & 13.72 \\
Silk I & $12.2^{\circ}$ & 2.67 & 8.11 & 10.16 \\
Amorphous & & 92.40 & 66.02 & 57.95
\end{tabular}

${ }^{a} A_{\mathrm{i}}$ is the related peak area of silk I, silk II structure $\left(i=12.2^{\circ}, 20.6^{\circ}\right.$, $\left.24.3^{\circ}\right), A$ is total peak area. 
gradually shifted to $1525 \mathrm{~cm}^{-1}$, reflecting a transition of random coil conformation to $\beta$-sheet conformation. The results of conformation transformation good agree with the observation of the contact angle.

\section{XRD analysis}

X-ray diffraction was used to further confirm FTIR observations, and the results are given in Fig. 7. The presence of many distinct peaks at $10-30^{\circ}$ in the XRD patterns (Fig. 7A) was results of raw $\mathrm{DOX} \cdot \mathrm{HCl}$ and $\mathrm{CUR}$, which showed that the $\mathrm{DOX} \cdot \mathrm{HCl}$ and CUR were crystalline. ${ }^{37,38} \mathrm{Fig} .7 \mathrm{~B}(\mathrm{~b})$, the dual drug loaded RSF nanofibers showed broad humps pattern, absence of any distinct diffraction peaks, indicating that CUR and $\mathrm{DOX} \cdot \mathrm{HCl}$ were encapsulated in amorphous forms in the fibers. After water-annealing at temperature of 45 and $60{ }^{\circ} \mathrm{C}$, the patterns of the dual drug loaded RSF fibers cannot be seen the related characteristic diffraction peaks of CUR and
$\mathrm{DOX} \cdot \mathrm{HCl}$, but just can be seen the related silk I and silk II characteristic peaks of RSF nanofibers. With the temperature increased from 45 to $60{ }^{\circ} \mathrm{C}$, the central peak at $20.6^{\circ}$ (silk II) was narrow, and the peaks at $12.2^{\circ}$ (silk I) and at $24.3^{\circ}$ (silk II) appeared, reflecting a transition of amorphous structure to silk I and silk II structure. The contents of the amorphous and crystalline of these RSF nanofibers were calculated through peak separation by Peak Fit v 4.12 software, the results shows in Table 1.

Before the treatment, the content of amorphous of drugloaded RSF nanofibers was $92.4 \%$. After the treatment, the contents of crystalline silk II of that were increased from 25.87 to $31.89 \%$, and the content of crystalline silk I of that were increased from 8.11 to $10.16 \%$. Water-annealing treatment could induced a structure transition of RSF nanofibers from amorphous to silk II structure, but the silk I structure were still existed in RSF nanofibers at the processes condition of this paper, which good agreed with the previous FTIR results.
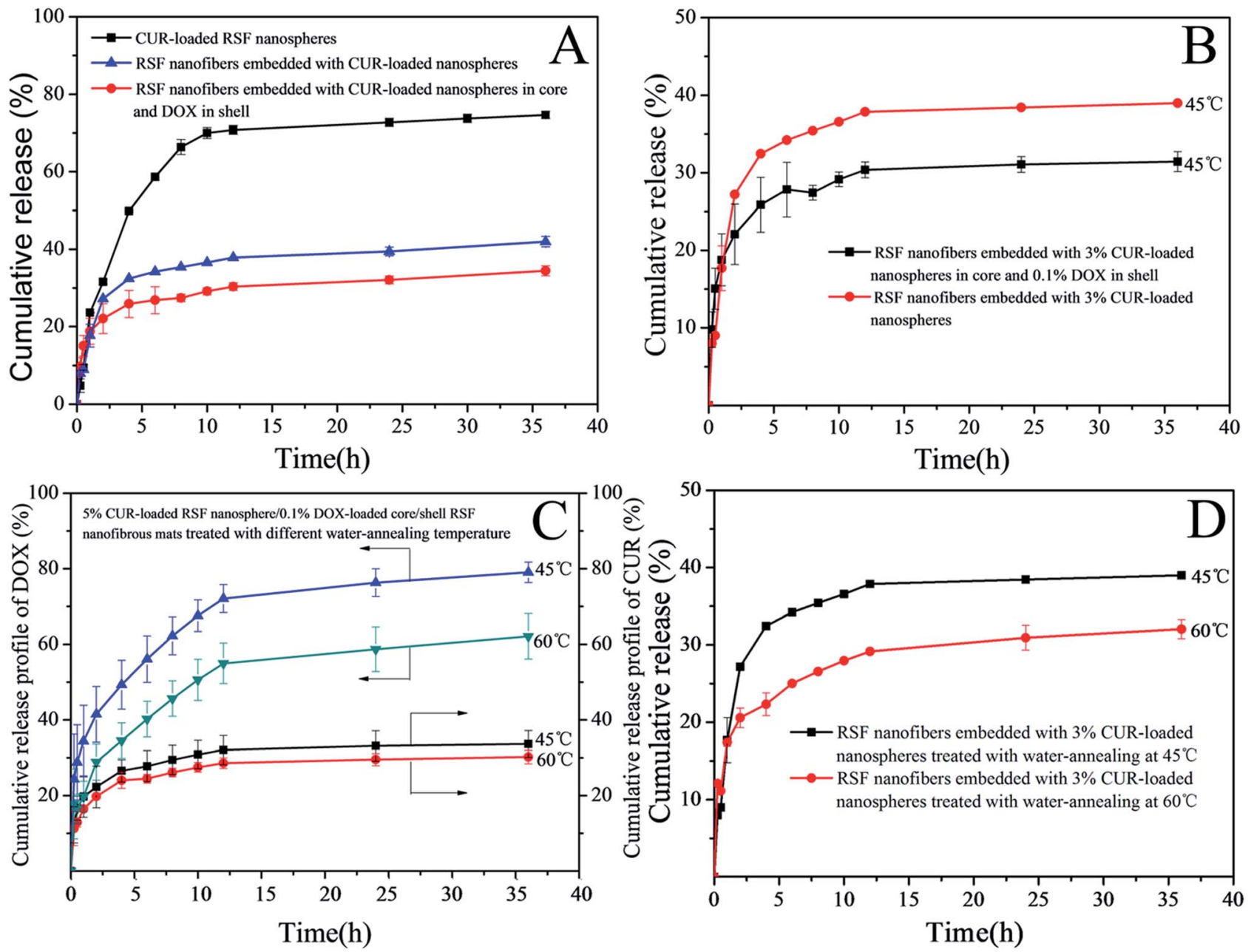

Fig. 8 The release profiles of drug loaded RSF nanofibrous mats: (A) the release of CUR from CUR-loaded RSF nanospheres, RSF nanofibers embedded with CUR-loaded nanospheres, and RSF nanofibers embedded with CUR-loaded nanospheres in core and DOX.HCl in shell, respectively; (B) the release of CUR from 3\% CUR-loaded nanospheres RSF nanofibers and 3\% CUR-loaded RSF nanospheres/0.1\% DOX.HClloaded core/shell RSF nanofibers treated with water-annealing at temperature of $45^{\circ} \mathrm{C} ;(\mathrm{C})$ the release of CUR and DOX from $5 \% \mathrm{CUR}$-loaded RSF nanospheres/0.1\% DOX. $\mathrm{HCl}$-loaded core/shell RSF nanofibers treated with water-annealing at temperature of 45 and $60{ }^{\circ} \mathrm{C}$, (D) the release of CUR from 3\% CUR-loaded nanospheres RSF nanofibers treated with water-annealing at temperature of 45 and $60{ }^{\circ} \mathrm{C}$, respectively. 


\section{Drug release study}

Fig. 8A shows the release profiles of CUR from CUR-loaded RSF nanospheres, RSF nanofibers embedded with CUR-loaded nanospheres, CUR-loaded RSF nanospheres/DOX $\cdot \mathrm{HCl}$-loaded core/shell RSF nanofibers RSF in release medium (PBS/ ethanol $=5: 5(\mathrm{v} / \mathrm{v}))$ at $37{ }^{\circ} \mathrm{C}$, respectively. It can be seen that there was relatively slow release process for RSF nanofibers embedded with CUR-loaded nanospheres and CUR-loaded RSF nanospheres/DOX·HCl-loaded core/shell RSF nanofibers compared to that of CUR-loaded RSF nanospheres in the first several hours. After the release process of $36 \mathrm{~h}$, it was found that CUR release rate, RSF nanofibers embedded with CUR-loaded nanospheres and CUR-loaded RSF nanospheres/DOX·HClloaded core/shell RSF nanofibers, was $41.99 \%$ and $34.43 \%$ respectively, whereas the CUR release rate of CUR-loaded RSF nanospheres was up to $74.66 \%$. In the case of drug loaded RSF nanofibers, apart from the barrier of the core layer, the CUR molecules had to permeate across the shell barrier of RSF nanofibers to arrive at the release medium. So the drug release rate is down to $41.99 \%$ and $34.43 \%$, respectively. This confirms that the RSF nanofibrous mats could provide a slow, long term release for a drug.

Fig. 8B shows the CUR release profiles of RSF nanofibers embedded with 3\% CUR-loaded nanospheres and 3\% CURloaded RSF nanospheres/0.1\% DOX·HCl-loaded core/shell RSF nanofibers in release medium of PBS/ethanol $=5: 5(\mathrm{v} / \mathrm{v})$ at $37^{\circ} \mathrm{C}$. In the early stage $(2 \mathrm{~h})$ of drug release, the release rate of CUR in the dual drug loaded core/shell nanofibers was faster than that of the single-drug loaded ones, which may be due to the thinning of the fiber diameter after the loading of $\mathrm{DOX} \cdot \mathrm{HCl}$ (the average diameter of dual-drug loaded core/shell fibers was $987 \mathrm{~nm}$ and single-drug loaded fibers was $1224 \mathrm{~nm}$ ). Thus, it has a large specific surface area and could contact with the outside medium adequately, and the diffusion of drug molecules into the medium was faster. In the mid and later stage of drug release, the release rate of CUR in the dual drug loaded nanofibers was slower than that of CUR in the single-drug loaded ones, and reached at $31.40 \%$ and $39.03 \%$ respectively. This may be due to the fact that dual drug loaded nanofibers has relatively higher drug density than that of the single-drug loaded ones and thus reduce the diffusion velocity of the water molecules to enter the fibers. These results suggested that the release behavior of CUR from dual drug loaded fibers might be affected by the fiber diameter and drug density. ${ }^{39}$

In order to understand the effect of crystal content of RSF nanofibers on drug release, we tested the drug release behavior of single and dual drug loaded RSF nanofibers treated with water-annealing at different temperature and the drug release profiles are illustrated in Fig. $8 \mathrm{C}$ and D. It can be seen that (Fig. 8C) the release level of DOX from 5\% CUR-loaded nanospheres/0.1\% DOX·HCl-loaded RSF core/shell nanofibers was higher than the release level of CUR from the same one. This is because DOX· $\mathrm{HCl}$ was loaded in shell layer of nano-fibers and CUR was loaded in nanospheres which were embedded in core layer of nanofibers. During the release process of the drug, the diffusion front of DOX was across the shell layer of RSF nanofibers to the release medium, while the diffusion front of CUR had to migrate through the coating layer of nanosphere to the shell layer of RSF nanofibers, and then into the release medium. Meanwhile, from the point of view of the first layer barrier of drug diffusion, the concentration gradient of DOX between the shell layer of RSF nanofibers and the unsaturated release medium was higher than that of CUR between the coating layer of nanosphere and the shell layer of RSF nanofibers. So the release rate of DOX was higher than that of CUR.

Compared Fig. 8C with Fig. 8D, it can be found that the release profiles of CUR from nanofibers followed the similar trend, whether it's from a single drug or dual drug nanofibers. That is, the release rate of drug from nanofibers treated at $45{ }^{\circ} \mathrm{C}$ was relatively faster than that of drug from RSF nanofibers treated at $60{ }^{\circ} \mathrm{C}$. This was because nanofibers treated at $45{ }^{\circ} \mathrm{C}$ contained low crystal content $(25.87 \%$ silk II, $8.11 \%$ silk I, schematic diagram in Fig. 9B), while nanofibers treated at $60{ }^{\circ} \mathrm{C}$ contained high crystal content (31.89\% silk II, $10.16 \%$ silk I, schematic diagram in Fig. 9C).

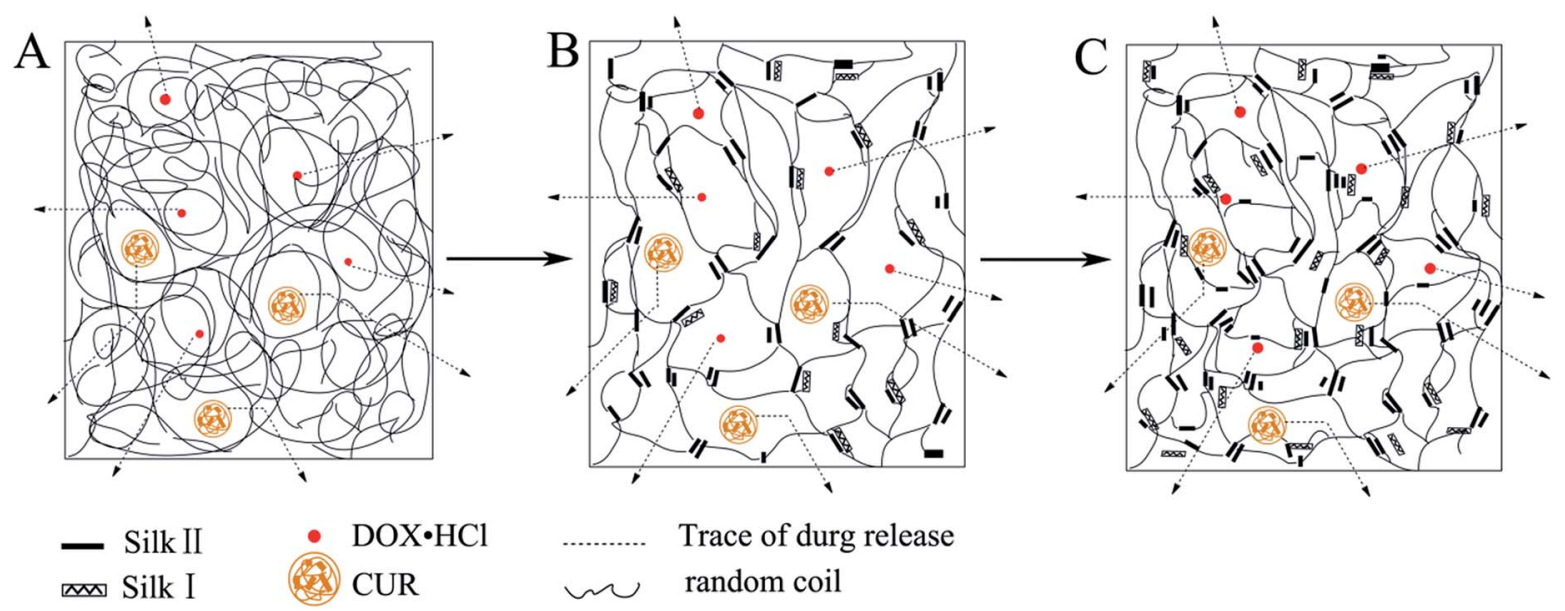

Fig. 9 Schematic diagram of drug release simulation: (A) schematic diagram of CUR and DOX.HCl from untreatment sample (B) schematic diagram of CUR and DOX. $\mathrm{HCl}$ from low content of silk II crystal (C) schematic diagram of CUR and DOX. $\mathrm{HCl}$ from high content of silk II crystal. 
Table 2 Parameters obtained by fitting the drug release profile of dual drug loaded RSF nanofibers treated with water-annealing at different temperature

\begin{tabular}{lccc}
\hline Samples release curve & $k$ & $n$ & $R^{2}$ \\
\hline $\begin{array}{l}\text { CUR release rate of dual } \\
\text { drug nanofibers treated at } 45{ }^{\circ} \mathrm{C}\end{array}$ & 34.851 & 0.278 & 0.997 \\
$\begin{array}{l}\text { CUR release rate of dual } \\
\text { drug nanofibers treated at } 60{ }^{\circ} \mathrm{C}\end{array}$ & 20.904 & 0.380 & 0.996 \\
$\begin{array}{l}\text { DOX } \cdot \mathrm{HCl} \text { release rate of } \\
\text { dual drug nanofibers treated at } 45{ }^{\circ} \mathrm{C}\end{array}$ & 19.482 & 0.202 & 0.998 \\
$\begin{array}{l}\text { DOX·HCl release rate of } \\
\text { dual drug nanofibers treated at } 60{ }^{\circ} \mathrm{C}\end{array}$ & 15.918 & 0.242 & 0.996 \\
& & &
\end{tabular}

When drug loaded nanofibers was submerged in release medium, water molecules penetrated into RSF fibers, RSF matrix was swollen the drug was diffusion from nanofibers. The crystalline region of RSF matrix could limit the swelling ratio of the matrix and the diffusion rate of drug from nanofibers, thus the presence of low crystal content in nanofibers allowed greater water permeation and leaded to relatively higher release rate of drug. The schematic diagram of drug diffusion from nanofibers is showed in Fig. 9. By controlling the content of drug loaded and crystal content, we can achieve the purpose of control the dual drug release rate.

\section{Study on the release mechanism}

In order to study drug-release mechanisms, we fitted the drug release profile in Fig. 8C. The values of release coefficient $k$, diffusional exponent $n$, and correlation coefficient $R^{2}$ are summarized in Table 2. The $R^{2}$ value indicates that the Korsmeyer-Peppas model is best suited for the release kinetics. Research shows that the release exponent of thin film, cylinder and sphere is $0.5,0.45$ and 0.43 according to Fickian diffusion mechanism, respectively. ${ }^{40}$ For the drug-loaded nanofibers, the $n$ value was within the limiting value of 0.45 in all the cases, the release exponent is a clear indication that Fickian model could be the principal release mechanism for drug.

\section{Conclusions}

In the present work, dual polarities drugs, curcumin (CUR, hydrophobic drug, loading in nanospheres) and doxorubicin hydrochloride (DOX $\cdot \mathrm{HCl}$, hydrophilic drug) were fabricated in aqueous-based regenerated silk fibroin nanofibers with a simple, colloid-electrospinning technique. The content of CUR-loaded nanospheres affected the fiber diameter and distribution of drug loaded nanofiber. TEM and fluorescence microscope confirmed CUR-loaded nanospheres core phase and $\mathrm{DOX} \cdot \mathrm{HCl}$ shell phase were dispersed and encapsulated in nanofibers. FTIR spectra demonstrated that RSF nanofibers had good compatibility with $\mathrm{CUR}$ and $\mathrm{DOX} \cdot \mathrm{HCl}$ as a result of hydrogen bonds forming. XRD tests verified that dual drug components in the nanofibers were presented in an amorphous state and the crystal content of RSF nanofibers could be tuned by water-annealing process at different temperature. In vitro dissolution tests showed that CUR-loaded nanospheres/ DOX $\cdot$ HCl-loaded RSF core/shell nanofibers exhibited dual drug release profiles and achieved a sustained release, while the amount of drug release in core or shell phase of nanofibers was tunable by controlling the drug-loaded content and crystal content of nanofibers. The release kinetics study showed that the mechanism of drug release belonged to Fickian model. This drug delivery system gives the possibility to fabricate multi-drug delivery systems that needs to be achieve synergistic effect and seems to be a promising candidates for use in tissue engineering and regenerative medicine.

\section{Conflicts of interest}

The authors declare there is no conflict of interest.

\section{Acknowledgements}

The authors acknowledge financial support from National Natural Science Foundation of China (51303124) and Shanxi Scholarship Council of China (Grant No. 2016-024).

\section{Notes and references}

1 T. J. Sill and H. A. V. Recum, Biomaterials, 2008, 29, 19892006.

2 S. F. Chou, D. Carson and K. A. Woodrow, J. Controlled Release, 2015, 220, 584-591.

3 D. S. Katti, K. W. Robinson, F. K. Ko and C. T. Laurencin, J. Biomed. Mater. Res., Part B, 2004, 70, 286-296.

4 M. Zamani, M. Morshed, J. Varshosaz and M. Jannesari, Eur. J. Pharm. Biopharm., 2010, 75, 179-185.

5 S. UdayKumar, I. Matai, P. Dubey, B. Bhushan, A. Sachdev and P. Gopinath, RSC Adv., 2014, 4, 38263-38272.

6 E. Wenk, H. P. Merkle and L. Meinel, J. Controlled Release, 2011, 150, 128-141.

7 Y. H. Shan, L. H. Peng, X. Liu, X. Chen, J. Xiong and J. Q. Gao, Int. J. Pharm., 2015, 479, 291-301.

8 L. Fan, H. Wang, K. Zhang, Z. Cai, C. He, X. Sheng and X. Mo, RSC Adv., 2012, 2, 4110-4119.

9 W. Liu, Y. Wang, J. Yao, Z. Shao and X. Chen, Mater. Lett., 2016, 178, 31-34.

10 M. Beck-Broichsitter, M. Thieme, J. Nguyen, T. Schmehl, T. Gessler, W. Seeger, S. Agarwal, A. Greiner and T. Kissel, Macromol. Biosci., 2010, 10, 1527-1535.

11 Z. Li, L. Song, X. Huang, H. Wang, H. Shao, M. Xie, Y. Xu and Y. Zhang, RSC Adv., 2015, 5, 16748-16758.

12 D. G. Yu, G. R. Williams, X. Wang, X. K. Liu, H. L. Li and S. W. A. Bligh, RSC Adv., 2013, 3, 4652-4658.

13 Q. Liu, J. Huang, H. Shao, L. Song and Y. Zhang, RSC Adv., 2016, 6, 7683-7691.

14 L. Tian, M. P. Prabhakaran, J. Hu, M. Chen, F. Besenbacherb and S. Ramakrishna, RSC Adv., 2015, 5, 49838-49848.

15 C. Ball, S. F. Chou, Y. Jiang and K. A. Woodrow, Mater. Sci. Eng., C, 2016, 63, 117-124. 
16 S. K. Murase, L. P. Lv, A. Kaltbeitzel, K. Landfester, L. J. del Valle, R. Katsarava, J. Puiggali and D. Crespy, $R S C A d v$, 2015, 5, 55006-55014.

17 L. Jorgensen, K. Qvortrup and I. S. Chronakis, RSC Adv., 2015, 5, 53644-53652.

18 J. Zhu, W. Huang, Q. Zhang, S. Ling, Y. Chen and D. L. Kaplan, Materials, 2016, 9, 221.

19 P. Wen, K. Feng, H. Yang, X. Huang, M. Zong, W. Lou, N. Li and H. Wu, Carbohydr. Polym., 2017, 169, 157-166.

20 D. Crespy, K. Friedemann and A. M. Popa, Macromol. Rapid Commun., 2012, 33, 1978-1995.

21 S. Jiang, L. P. Lv, K. Landfester and D. Crespy, Acc. Chem. Res., 2016, 49, 816-823.

22 S. Jiang, L. Lv, Q. Li, J. Wang, K. Landfester and D. Crespy, Nanoscale, 2016, 8, 11511-11517.

23 B. B. Aggarwal and K. B. Harikumar, Int. J. Biochem. Cell Biol., 2009, 41, 40-59.

24 S. C. Gupta, S. Patchva and B. B. Aggarwal, AAPS J., 2013, 15, 195-218.

25 C. Ramachandran, E. Escalon, Z. Khatib and S. Melnick, Cancer Res., 2007, 67, 4786.

26 W. Ma, Q. Guo, Y. Li, X. Wang, J. Wang and P. Tu, Eur. J. Pharm. Biopharm., 2016, 112, 209-223.

27 J. Zhu, Y. Zhang, H. Shao and X. Hu, Polymer, 2008, 49, 28802885.

28 S. Yu, W. Yang, S. Chen, M. Chen, Y. Liu, Z. Shao and X. Chen, RSC Adv., 2014, 4, 18171-18177.
29 C. Zheng, G. Chen and Z. Qi, Plasma Chem. Plasma Process., 2012, 32, 629-642.

30 T. Yucel, M. L. Lovett and D. L. Kaplan, J. Controlled Release, 2014, 190, 381-397.

31 X. Hu, K. Shmelev, L. Sun, E. K. Gil, S. H. Park, P. Cebe and D. L. Kaplan, Biomacromolecules, 2011, 12, 1686-1696.

32 D. Brahatheeswaran, A. Mathew, R. G. Aswathy, Y. Nagaoka, K. Venugopal, Y. Yoshida, T. Maekawa and D. Sakthikumar, Biomed. Mater., 2012, 7, 045001.

33 Z. Mai, J. Chen, T. He, Y. Hu, X. Dong, H. Zhang, W. Huang, F. Ko and W. Zhou, RSC Adv., 2017, 7, 1724-1734.

34 S. Wu, X. Zhao, Y. Li, Q. Du, J. Sun, Y. Wang, X. Wang, Y. Xia, Z. Wang and L. Xia, Materials, 2013, 6, 2026-2042.

35 B. Surnar and M. Jayakannan, ACS Biomater. Sci. Eng., 2016, 2, 1926-1941.

36 V. Alinejad, M. H. Somi, B. Baradaran, P. Akbarzadeh, F. Atyabi, H. Kazerooni, H. S. Kafil, L. A. Maleki, H. S. Mansouri and M. Yousefi, Biomed. Pharmacother., 2016, 83, 229-240.

37 R. Fan, X. Li, J. Deng, X. Gao, L. Zhou, Y. Zheng, A. Tong, X. Zhang, C. You and G. Guo, Sci. Rep., 2016, 6, 28373.

38 J. Wang, L. Li, L. Wu, B. Sun, Y. Du, J. Sun, Y. Wang, Q. Fu, P. Zhang and Z. He, Eur. J. Pharm. Sci., 2017, 99, 185-192.

39 Y. Wang, B. Wang, W. Qiao and T. Yin, J. Pharm. Sci., 2010, 99, 4805-4811.

40 J. Siepmann and F. Siepmann, Int. J. Pharm., 2008, 364, 328343. 